\title{
Bulk ionization potentials and band alignments from three-dimensional periodic calculations as demonstrated on rocksalt oxides
}

\author{
Andrew J. Logsdail, ${ }^{1, *}$ David O. Scanlon,,${ }^{1,2}$ C. Richard A. Catlow, ${ }^{1}$ and Alexey A. Sokol ${ }^{1, \dagger}$ \\ ${ }^{1}$ University College London, Kathleen Lonsdale Materials Chemistry, Department of Chemistry, 20 Gordon St., \\ London WC1H OAJ, United Kingdom \\ ${ }^{2}$ Diamond Light Source Ltd., Diamond House, Harwell Science and Innovation Campus, Didcot, Oxfordshire OX11 ODE, United Kingdom \\ (Received 13 November 2013; revised manuscript received 26 August 2014; published 6 October 2014)
}

\begin{abstract}
The position of the band edges of a material plays a key role in determining the properties for a range of applications, but fundamental band bending is an interface-dependent property that cannot be quantified without knowledge of bulk electron energy levels. We present a method for calculating the bulk position of the valence band maximum, and therefore the bulk ionization potential, from periodic plane wave calculations as shown for a range of rocksalt ionic oxides. We demonstrate that, for the popular "slab alignment" technique, explicit consideration of any surface induced electronic polarization is necessary to calculate accurate bulk ionization potentials. Our proposed method to quantify these surface effects, using polarizable-shell based interatomic potentials, is very computationally affordable, and our updated slab alignment method yields much improved agreement with the available experimental data.
\end{abstract}

DOI: 10.1103/PhysRevB.90.155106

PACS number(s): 71.15.Dx, 31.15.A-, 71.15.Mb, 71.20.-b

\section{INTRODUCTION}

Knowledge of ionization potentials, fundamental band bending, and electronic band alignment is crucial to our understanding of wide-ranging physical phenomena and chemical processes at the surfaces and interfaces of different materials [1]. A number of heterojunction alignment schemes have been advanced based on surface spectroscopic, electrical contact, or electrochemical measurements [1-7], which were also supported by electronic structure calculations, typically using density functional theory (DFT) [7-12]. Based on such schemes, complemented by studies of defect energetics, a concept of a fundamental charge neutrality level has been proposed that is common in all materials and exploited to interpret or predict defect properties and/or response to radiation [13-15]. The schemes, however, differ significantly in their positioning of the charge neutrality level with respect to vacuum or mutual band alignment. In contrast, the vacuum level itself is common to all materials by definition; it can serve as a common source or sink of charge for all finite samples. Therefore, establishing a robust and reliable methodology that will let us access this level from commonly employed $a b$ initio techniques is necessary.

Currently, there are several computational approaches to the calculation of bulk ionization potentials and band alignment; each, however, has inherent difficulties. Indeed, we aspire to quantify a local property of the bulk system that is assumed to be independent of surface termination, and therefore periodic boundary conditions (PBC) are applied [16] to a unit cell for calculation purposes, most commonly in the context of KohnSham equations $[17,18]$, where the energy reference is a result of particular implementation of lattice sums [19] and, usually, is not trivial to assess. In one approach, physical models of real interfaces are constructed, thus providing directly the band structure across the interface [10,20-23]. Upon

\footnotetext{
*a.logsdail@ucl.ac.uk

†a.sokol@ucl.ac.uk
}

formation of the interface between two different materials, a dipole is necessarily formed, which results in an offset in the electrostatic potential, for which in practice it is difficult to obtain a converged value [14]. An alternative method, which is less commonly applied, employs two-dimensional periodic slab models and provides direct access to a common reference level as an asymptote in vacuum, e.g., as implemented in CRYSTAL [24]. Here, the problem arises in turn from a dipole formed on surface termination, but at least now it depends on the properties of one material only, and the potential offset is the real physical origin of surface band bending. However, the two-dimensional (2D) lattice sums are not well suited for plane-wave methods, implemented in such popular codes as CPMD [25], CASTEP [26], and VASP [27,28]. To access the vacuum level from plane-wave calculations, a periodic slab model is commonly employed, akin to the 2D slab discussed above; however, a large interslab vacuum layer is created. The middle of the slab approximates the bulk, whereas the middle of the empty layer represents vacuum [29-34].

In this work, we demonstrate that, due to the lack of consideration for surface polarization, this approach results in errors even for the simplest rocksalt structured II-VI ionic oxides, themselves of scientific interest due to their applications as catalytic substrates $[35,36]$ and characterizable electronic properties [37,38], some of which are quite unusual, e.g., negative electron affinity, or work function [39]. Furthermore, the stability of the (100) surfaces for these materials [40] means that experimental surface measurements can be compared to simulations without need for consideration of complex surface restructuring. We propose a scheme for calculating the position of the vacuum potential for bulk materials that takes advantage of the intuitive strengths of the previous method, but also takes into account the potential offset incurred through polarization effects, accounting for the electronic redistribution at the slab terminating surfaces in a semiclassical approximation. Our approach results in a substantial improvement in comparison to experiment, while being only slightly more computationally expensive. 


\section{METHODOLOGY}

\section{A. Bulk ionization potential}

By convention, the vacuum potential, $V_{\text {vac }}$, is the potential of an electron far removed from the bulk, and is set to zero. The difference between the highest occupied one-electron energy level of a system and the vacuum level, $-e V_{\mathrm{vac}}$, is considered to be the first ionization potential of a system via Koopman's theorem [41]; while this approximation is not strictly valid for the Kohn-Sham formalism, the errors in the accuracy of resulting electronic energies have been shown to be small for bound states [42]. The highest occupied energy level $\left(\epsilon_{\mathrm{VBM}}\right)$ is referred to as the energy of the valence band maximum (VBM) in bulk materials, and therefore the first ionization potential of the bulk can be defined as the energy required to move an electron from the VBM to vacuum [43]:

$$
I_{\mathrm{VBM}}^{\mathrm{b}}=-e V_{\mathrm{vac}}-\epsilon_{\mathrm{VBM}}^{\mathrm{b}}=-\epsilon_{\mathrm{VBM}}^{\mathrm{b}},
$$

where $\mathrm{b}$ denotes the bulk.

In gas-phase calculations, using for example localized basis sets, periodicity is not applied, and the concept of the vacuum potential far removed from a molecule is easily understood. However, the inherent periodicity of a plane-wave basis means that in periodic calculations one must create a vacuum region where $V_{\text {vac }}$ can be accessed. Typically, this is achieved by creating a surface slab "supercell," where a thick slab layer represents the bulk material, and a large interslab vacuum space is included to allow assessment of $V_{\text {vac }}$. In this case, however, the electrostatic potential does not disappear in the middle of the vacuum region $\left(V_{\mathrm{bg}}^{\mathrm{s}}\right.$, where bg signifies the background level, and s signifies surface), but may remain finite [30,32] due to both the slow convergence of the 3D lattice sums with respect to the vacuum-layer width and/or the effects of surface band bending. However, $V_{\mathrm{bg}}^{\mathrm{s}}$ is analogous to $V_{\text {vac }}$, and so the two may be aligned with a shift in energy equal in magnitude to $e V_{\mathrm{bg}}^{\mathrm{s}}$, with $e$ being the electron charge. By shifting all the electron energies by the same quantity, the "absolute" electron energies for a slab can therefore be obtained [29,32], as illustrated in Fig. 1.

If the slab layer is thick enough then the atoms in the middle of the slab may be likened to being in a bulk environment; thus, it is possible to relate the energy levels for the central atoms of the slab to the energy levels of the same atoms in the bulk. Assuming that the lowest energy core states (e.g., $1 s$ ) on atoms in the middle of the slab are unperturbed by surface effects, due to their explicit localized nature, one can take such core states in the slab and calculate the difference between their energy $\left(\epsilon_{\mathrm{c}}^{\mathrm{s}}\right.$, where $\mathrm{c}$ signifies the core level) and the energy of the same quantum state in the bulk $\left(\epsilon_{\mathrm{c}}^{\mathrm{b}}\right)$ [44]:

$$
\Delta \epsilon_{\mathrm{c}}=\epsilon_{\mathrm{c}}^{\mathrm{b}}-\epsilon_{\mathrm{c}}^{\mathrm{s}} .
$$

One can then define the ionization potential of the reference $1 s$ core state in the slab model $\left(I_{\mathrm{c}}\right)$ as the energy required to take an electron from the core level $\left(\epsilon_{\mathrm{c}}^{\mathrm{s}}\right)$ to the midvacuum layer $\left(V_{\mathrm{bg}}^{\mathrm{s}}\right)$; by convention this approximation then equates $I_{\mathrm{c}}$ to the ionization potential of the corresponding state in the bulk as [29]

$$
\begin{aligned}
& I_{\mathrm{c}}^{\mathrm{s}}=-e V_{\mathrm{bg}}^{\mathrm{s}}-\epsilon_{\mathrm{c}}^{\mathrm{s}}, \\
& I_{\mathrm{c}}^{\mathrm{b}}=-e V_{\mathrm{bg}}^{\mathrm{s}}-\epsilon_{\mathrm{c}}^{\mathrm{b}}+\Delta \epsilon_{\mathrm{c}}=I_{\mathrm{c}}^{\mathrm{s}},
\end{aligned}
$$

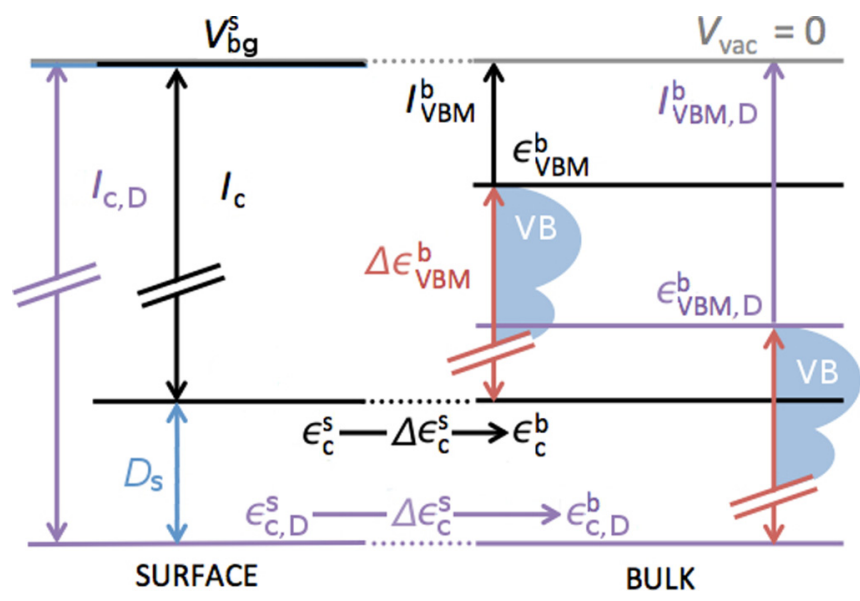

FIG. 1. (Color online) Diagram of vacuum alignment methods: $I_{\mathrm{c}}$ (black) is the difference between $-e V_{\mathrm{bg}}^{\mathrm{s}}$ and $\epsilon_{\mathrm{c}}^{\mathrm{s}}$, and $\epsilon_{\mathrm{c}}^{\mathrm{b}}$ is the position of the core levels in bulk using this method; $I_{\mathrm{c}, \mathrm{D}}$ (purple) is the difference between $-e V_{\mathrm{bg}}^{\mathrm{s}}$ and $\epsilon_{\mathrm{c}}^{\mathrm{s}}$ with $D_{\mathrm{s}}$ considered explicitly, and $\epsilon_{\mathrm{c}, \mathrm{D}}^{\mathrm{b}}$ is the position of the core levels in bulk using this method. $\Delta \epsilon_{\mathrm{VBM}}^{\mathrm{b}}$ (red) is the difference in energy between the core and valence levels in bulk, used to position the valence band maximum (VBM) by each method ( $\epsilon_{\mathrm{VBM}}^{\mathrm{b}}$ and $\left.\epsilon_{\mathrm{VBM}, \mathrm{D}}^{\mathrm{b}}\right) ; V B$ (white) is the valence band; $V_{\text {vac }}$ (gray) is the vacuum level. $I_{\mathrm{VBM}}$ is therefore the first ionization potential of the bulk, calculated as the difference between $-e V_{\text {vac }}$ and $\epsilon_{\mathrm{VBM}}^{\mathrm{b}}\left(\right.$ black) and $\epsilon_{\mathrm{VBM}, \mathrm{D}}^{\mathrm{b}}$ (purple) and labeled as $I_{\mathrm{VBM}}^{\mathrm{b}}$ and $I_{\mathrm{VBM}, \mathrm{D}}^{\mathrm{b}}$, respectively.

and from this the states in the bulk system can be aligned to the vacuum level by shifting all the energy levels in the bulk by $\Delta \epsilon_{\mathrm{c}}$. This therefore allows an absolute natural alignment of important bulk electron energies, such as $\epsilon_{\mathrm{VBM}}$, for different materials at the DFT level as the first ionization potential can now be defined as:

$$
I_{\mathrm{VBM}}^{\mathrm{b}}=-e V_{\mathrm{bg}}^{\mathrm{s}}-\epsilon_{\mathrm{VBM}}^{\mathrm{b}}+\Delta \epsilon_{\mathrm{c}} .
$$

For a full understanding of surface properties, including surface band bending effects from different surfaces and/or interfaces, $I_{\mathrm{c}}^{\mathrm{b}}$ provides a common reference energy. However, for a slab model with a fixed bulk geometry, introducing a surface will alter the electronic properties of a system: the additional electronic degrees of freedom will lead to electrons migrating to or away from the vacuum environment, resulting in the formation of surface multipoles that create an inherent offset in the electrostatic potential in a slab $[45,46]$. Therefore, we outline below a method that allows evaluation of surface polarization effects, which effectively recovers the surface independent nature of the alignment of bulk levels and thus offers the ability to calculate bulk ionization potentials; and only then can one begin to consider separately interfacedependent effects such as fundamental band bending. We note that the slab models could be geometry optimized for the calculation of surface specific properties, and then $\epsilon_{\mathrm{VBM}}^{\mathrm{s}}$ for a final geometry can be directly aligned with $V_{\mathrm{bg}}^{\mathrm{s}}$ to give a valid absolute electron energy level for this surface only. The geometry optimized surfaces could also be used to assess bulk energy levels; however, the analysis is complicated by the additional optimization of nuclear coordinates, which may 
lead to increases in surface polarization that can further shift $\epsilon_{\mathrm{c}}^{\mathrm{s}}$ [44]. Therefore, we emphasize here that the simplest method to assess $\epsilon_{\mathrm{c}}^{\mathrm{s}}$ accurately is by fixing the slab atomic structure to that of the bulk geometry.

In an ionic material, the shift in energy levels upon surface termination is correlated with changes in the underlying electrostatic potential; more specifically, electron redistribution will directly influence the electrostatic potential through the formation of surface multipoles. Therefore, changes in $\epsilon_{\mathrm{c}}^{\mathrm{s}}$ can be quantified by calculating the change in the electrostatic potential in the middle of the slab $\left(\Delta V_{\mathrm{c}}^{\mathrm{s}}\right)$ and in the middle of the vacuum gap ( $\Delta V_{\mathrm{bg}}^{\mathrm{s}}$ ) due to electron redistribution:

$$
\begin{gathered}
\Delta V_{\mathrm{c}}^{\mathrm{s}}=V_{\mathrm{c}}^{\mathrm{s}, \text { relax }}-V_{\mathrm{c}}^{\mathrm{s}}, \\
\Delta V_{\mathrm{bg}}^{\mathrm{s}}=V_{\mathrm{bg}}^{\mathrm{s}, \text { relax }}-V_{\mathrm{bg}}^{\mathrm{s}},
\end{gathered}
$$

where $V_{\mathrm{c}}^{\mathrm{s}}$ and $V_{\mathrm{bg}}^{\mathrm{s}}$ [as defined for Eq. (3)] are the electrostatic potentials before electron redistribution, and $V_{\mathrm{c}}^{\mathrm{s} \text {,relax }}$ and $V_{\mathrm{bg}}^{\mathrm{s} \text {,relax }}$ are the electrostatic potentials after electronic relaxation. With the nuclear coordinates fixed, an increase in $\Delta V_{\mathrm{c}}^{\mathrm{s}}$ indicates electron redistribution towards the surface, while a decrease results from electron redistribution towards the center of the slab. So in the absence of polarization, as in the bulk environment, $\Delta V_{\mathrm{bg}}^{\mathrm{s}}=\Delta V_{\mathrm{c}}^{\mathrm{s}}=0$. We can therefore define the surface polarization correction as the multipolar shift, $D_{\mathrm{s}}$ :

$$
D_{\mathrm{s}}=e\left(\Delta V_{\mathrm{bg}}^{\mathrm{s}}-\Delta V_{\mathrm{c}}^{\mathrm{s}}\right),
$$

where the dipolar parallel plate (planar) capacitor model is used to define the sign on $\Delta V_{\mathrm{c}}^{\mathrm{s}}$ and $\Delta V_{\mathrm{bg}}^{\mathrm{s}}$ : if an electronic dipole forms pointing in to the slab, with negative charge at the surface, then $\Delta V_{\mathrm{c}}^{\mathrm{s}}>0>\Delta V_{\mathrm{bg}}^{\mathrm{s}}$ [47]. This stabilizes $\epsilon_{\mathrm{c}}^{\mathrm{s}}$ relative to the $V_{\mathrm{bg}}^{\mathrm{s}}$, meaning that $I_{\mathrm{c}}^{\mathrm{s}}$ would be inflated. To remove this dipolar effect, therefore, the polarization free environment must be recreated, as achieved by subtracting out the positive shift in electrostatic potential for the central atoms in the slab, $-e \Delta V_{\mathrm{c}}^{\mathrm{s}}$, and by adding $e \Delta V_{\mathrm{bg}}^{\mathrm{s}}$ to remove the negative shift in $V_{\mathrm{bg}}^{\mathrm{s}}$.

$D_{s}$ can then be combined with $I_{\mathrm{c}}^{\mathrm{b}}$ to provide an improved assessment of the bulk ionization potentials:

$$
I_{\mathrm{c}, \mathrm{D}}^{\mathrm{b}}=I_{\mathrm{c}}^{\mathrm{b}}+D_{\mathrm{s}},
$$

where $I_{\mathrm{c}}^{\mathrm{b}}$ is as defined in Eq. (4). Thus $I_{\mathrm{c}, \mathrm{D}}^{\mathrm{b}}$ is the bulk ionization potential for the core levels including the effects for surface polarization, and bulk energy levels must be shifted by $D_{\mathrm{s}}$, as well as $\Delta \epsilon_{\mathrm{c}}$, in order to align them correctly with the $V_{\text {vac }}$. This applies to all energy levels and thus the first ionization potential, as defined in Eq. (5), similarly becomes $I_{\mathrm{VBM}, \mathrm{D}}^{\mathrm{b}}=I_{\mathrm{VBM}}^{\mathrm{b}}+D_{\mathrm{s}}$.

\section{B. Computational details}

DFT calculations have been performed using a plane-wave basis set, as implemented in the software package VASP $[27,28]$, to obtain accurate electron energy levels. Only the valence electronic configurations were calculated explicitly, with interactions between the core and valence electrons described using the PAW method [48]. The valence electronic configurations for $\mathrm{O}\left(2 s^{2} 2 p^{4}\right), \mathrm{Mg}\left(2 p^{6} 3 s^{2}\right), \mathrm{Ca}\left(3 s^{2} 3 p^{6} 4 s^{2}\right)$, $\mathrm{Sr}\left(4 s^{2} 4 p^{6} 5 s^{2}\right)$, and $\mathrm{Ba}\left(5 s^{2} 5 p^{6} 6 s^{2}\right)$ all included semicore states. A plane-wave kinetic energy cutoff of $500 \mathrm{eV}$ and a con- verged Monkhorst-Pack grid [49] across the first Brillouin zone with k-points spacing of $0.04 \AA^{-1}$ or denser have been used throughout. The sole unit cell parameter for cubic rocksalts, $a_{0}$, was optimized using the analytical stress tensor, and the plane waves were adjusted between geometry optimization steps to remove Pulay stress [50]. Structural convergence was achieved when the forces on all atoms were less than $0.01 \mathrm{eV}^{-1}$. We have used the PBE [51] and PBESol [52] exchange-correlation (XC) functionals, which are both examples of the generalized gradient approximation (GGA), as well as their nonlocal hybrid counterparts, namely PBE0 [53] and PBESol0, wherein $25 \%$ of Hartree-Fock exchange is combined with the GGA XC functionals $[53,54]$. High-frequency dielectric constants were calculated via linear response density functional perturbation theory at all four levels of theory [55], with > 200 unoccupied bands included in the calculation.

To represent surface termination, slab supercells were constructed from the optimized bulk unit cell with a (100) termination. The electronic structure in the middle of the slab should correctly reproduce the bulk electronic structure, and the slab is terminated with the (100) surface as this produces the lowest disturbance to the electronic structure, i.e. the fewest bonds are severed, has the lowest surface energy and, therefore, has the least effect on the electronic structure. Furthermore, a Tasker type 1 surface [56], with no residual polar moments, is preferably chosen; otherwise, higher order multipoles must be considered as contributing to $D_{\mathrm{s}}$. A minimum of eight atomic layers in the slab model and a vacuum layer of at least $18 \AA$ proved necessary to converge the electronic properties in the middle of the slab, with $\epsilon_{\mathrm{c}}^{\mathrm{s}}$ converged to $0.01 \mathrm{eV}$. The electronic structure of the slab was then calculated with DFT, with the atomic structure fixed in the bulklike arrangement [29], and $-e V_{\mathrm{bg}}^{\mathrm{s}}$ is taken to be the Hartree potential (energy density) in the middle of the vacuum layer, as illustrated in Fig. 2.

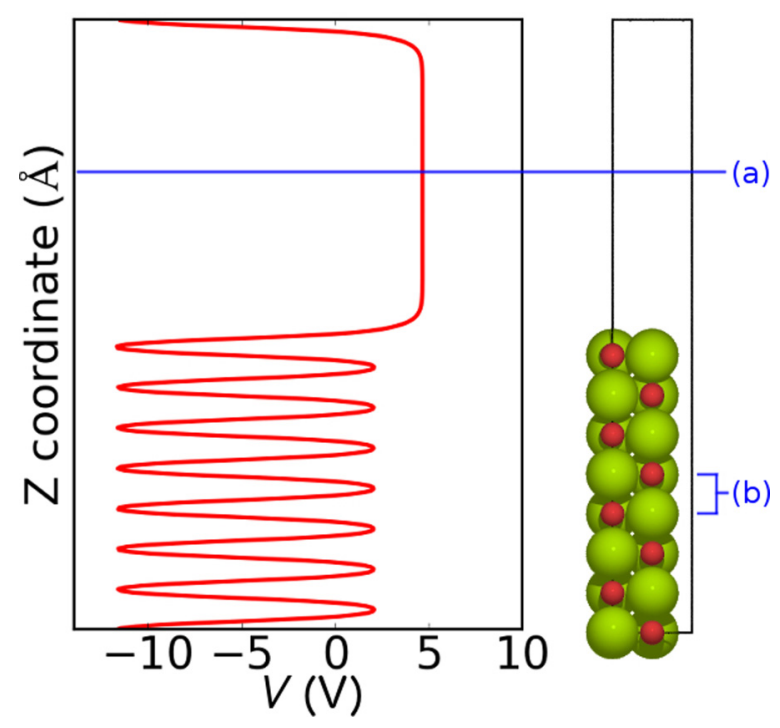

FIG. 2. (Color online) Example of an $\mathrm{MgO}$ surface unit cell (right) alongside a plot in red of the electrostatic potential normal to the slab surface, $V(z)$ (left). Highlighted in blue are (a) the midpoint of the interslab vacuum gap where $V_{\mathrm{bg}}^{\mathrm{s}}$ is evaluated and (b) the middle atoms of the slab structure, on which both $V_{\mathrm{c}}^{\mathrm{s}}$ and $\epsilon_{\mathrm{c}}^{\mathrm{s}}$ are evaluated. $\mathrm{Mg}$ and $\mathrm{O}$ atoms are shown in green and red, respectively. 
To calculate $\Delta V_{\mathrm{c}}^{\mathrm{s}}$ and $\Delta V_{\mathrm{bg}}^{\mathrm{s}}$, the effects of electronic relaxation in the supercell have been modeled as electronic polarization using the approach of Lewis and Catlow [57], in which an accurate account is given by the method of interatomic potentials coupled with a polarizable shell model as implemented in GULP [58]. In the polarizable shell model a harmonic energy contribution between the core and shell $\left(U_{\mathrm{c}-\mathrm{s}}\right)$ for atom $i$ is defined as

$$
U_{\mathrm{c}-\mathrm{s}, i}=\frac{1}{2} k_{i} r_{\mathrm{c}-\mathrm{s}, i}^{2},
$$

where $r_{\mathrm{c}-\mathrm{s}, i}$ is the distance between the core and shell of $i$, and the spring constant $k_{i}$ is defined as [58]

$$
k_{i}=Y^{2} / \alpha_{i},
$$

where $\alpha_{i}$ is the polarizability, and $Y$ is the shell charge of the respective ion. In this approach, cationic polarizabilities are assumed to be system independent and equal to those of gas phase ions (Lewis and Catlow have actually used the values of $\alpha_{i}$ originally tabulated by Pauling [59]), whereas the anionic polarizabilities are system and site dependent. The latter could be determined either by scaling the value of $\alpha_{i}$ taken from a system where such a value is known, using an approximate inverse proportionality of the polarizability and the Madelung site potential, or established empirically, for example by fitting the high-frequency dielectric constant or refractive indices. The potentials of Lewis and Catlow [57] were originally parametrized to experimentally observed properties, and we have reparametrized them to reproduce the bulk lattice $\left(a_{0}\right)$ and high-frequency bulk dielectric $[\varepsilon(\infty)]$ constants as obtained from DFT calculations to a high degree of accuracy $(0.0001 \%)$ for each XC functional. Furthermore, by taking into account that $\alpha_{i}$ is proportional to the site coordination number, we have reduced $k_{i}$ by a coordination factor of $n_{\mathrm{b}} / n_{i}$ for each surface terminating site, where $n_{\mathrm{b}}$ and $n_{i}$ are the coordination number for a bulk atom and atom $i$, respectively. The dependence of $k_{i}$ on the atomic coordination therefore accounts for changes in polarizability at surfaces and interfaces.

Using the XC-specific parametrized potentials, the slab models were subjected to electronic minimization, in which the atomic cores were fixed and only the polarizable shells were allowed to move; thus representing the relaxation of valence electrons within the DFT slab calculation. $\Delta V_{\mathrm{c}}^{\mathrm{s}}$ and $\Delta V_{\mathrm{bg}}^{\mathrm{s}}$ were calculated as the differences in the Madelung site potentials before and after shell relaxation at the oxygen core and the mid-vacuum gap, respectively.

\section{RESULTS AND DISCUSSION}

Presented in Table I are first ionization potentials (negative of the positions of the VBM), as calculated from a full band structure, relative to vacuum for metal oxides formed by group 2 elements of the Periodic Table $(\mathrm{MgO}, \mathrm{CaO}, \mathrm{SrO}$, and $\mathrm{BaO}$ ) aligned using Eqs. (3), (4), and (9), as well as the industrially relevant group 12 metal oxide CdO. Details of shell model parametrization and results from our DFT and interatomic potential based calculations are provided in the Appendices. As we have shown for our slab model, electron energies converge within four atomic layers $(\sim 10 \AA)$ and our calculated $I_{\mathrm{VBM}}$ can be compared directly to the experimental optical absorption spectra of mixed samples, where the
TABLE I. $I_{\mathrm{VBM}}^{\mathrm{b}}$ and $I_{\mathrm{VBM}, \mathrm{D}}^{\mathrm{b}}$ of rocksalt structured oxides, as calculated using Eqs. (3) and (9), respectively, to align the vacuum level. $I_{\mathrm{VBM}, \mathrm{D}}^{\mathrm{b}}$ is presented in bold, while $I_{\mathrm{VBM}}^{\mathrm{b}}$ is presented in italics. The bulk VBM were taken from $\Gamma$ for $\mathrm{MgO}, \mathrm{CaO}$, and $\mathrm{SrO}, M$ for $\mathrm{BaO}$, and $L$ for $\mathrm{CdO}$, respectively. For comparison, the first ionization potential as calculated from experimental optical absorption measurements is also presented where available $[39,61-$ 63], as well as an experimentally derived estimate for $\mathrm{CdO}$ from the work function [64-66].

\begin{tabular}{lccccc}
\hline \hline & Expt. & PBE & PBESol & PBE0 & PBESol0 \\
\hline $\mathrm{MgO}$ & 7.16 & $\mathbf{5 . 7 5}$ & $\mathbf{5 . 8 1}$ & $\mathbf{6 . 8 9}$ & $\mathbf{6 . 9 7}$ \\
& & $(4.79)$ & $(4.83)$ & $(6.04)$ & $(6.11)$ \\
$\mathrm{CaO}$ & 6.25 & $\mathbf{4 . 6 7}$ & $\mathbf{4 . 7 4}$ & $\mathbf{5 . 6 4}$ & $\mathbf{5 . 9 0}$ \\
& & $(4.29)$ & $(4.35)$ & $(5.47)$ & $(5.73)$ \\
$\mathrm{SrO}$ & 5.30 & $\mathbf{4 . 2 2}$ & $\mathbf{4 . 3 1}$ & $\mathbf{5 . 2 1}$ & $\mathbf{5 . 3 0}$ \\
& & $(4.06)$ & $(4.16)$ & $(5.27)$ & $(5.38)$ \\
$\mathrm{BaO}$ & & $\mathbf{3 . 3 7}$ & $\mathbf{3 . 3 2}$ & $\mathbf{4 . 1 4}$ & $\mathbf{4 . 1 7}$ \\
& & $(3.67)$ & $(3.66)$ & $(4.75)$ & $(4.80)$ \\
$\mathrm{CdO}$ & $6.04-6.78$ & & & $\mathbf{6 . 3 5}$ & $\mathbf{5 . 9 4}$ \\
& & & & $(5.69)$ & $(5.28)$ \\
\hline \hline
\end{tabular}

penetration depth of incident radiation is several orders of magnitude greater than the depth of any possible surface band bending (e.g., $\sim 25 \mu \mathrm{m}$ for $\mathrm{MgO}[60]$ ), and the (100) surfaces of these rocksalt structured materials are extremely stable with respect to other surfaces. Furthermore, surface rumpling on the (100) surface alters nuclear coordinates by at most $2 \%$ [40], and so it can be safely assumed that experimental optical measurements effectively evaluate $I_{\mathrm{VBM}}^{\mathrm{b}}$.

For all the XC functionals and materials considered, we observe changes in the absolute positioning of the VBM relative to $V_{\text {vac }}$ for the different methods of vacuum alignment. Turning first to the GGA XC functionals, for PBE and PBESol the $I_{\mathrm{VBM}}^{\mathrm{b}}$ (i.e., uncorrected for surface polarization) are significant underestimates of ca. $2 \mathrm{eV}$ for $\mathrm{MgO}$ and $\mathrm{CaO}$ when compared to $I_{\text {expt }}$, and $I_{\mathrm{VBM}}^{\mathrm{b}}$ of $\mathrm{SrO}$ is underestimated by ca. $1 \mathrm{eV}$. A better agreement is universally achieved for $I_{\mathrm{VBM} . \mathrm{D}}^{\mathrm{b}}$ (i.e., corrected for surface polarization), with the multipolar shift $D_{\mathrm{s}}$ increase/decrease of up to $0.98 / 0.34 \mathrm{eV}$ in the case of $\mathrm{MgO} / \mathrm{BaO}$, respectively. We also note that, when comparing $\mathrm{XC}$ functionals and alignment methods, PBESol provides a greater $I_{\mathrm{VBM}}^{\mathrm{b}}$ for the rock salt oxides formed from lighter cations $(\mathrm{Mg}, \mathrm{Ca}, \mathrm{Sr})$, while PBE gives a marginally greater $I_{\mathrm{VBM}}^{\mathrm{b}}$ for the heavier Ba cation. All GGA calculations, however, significantly underestimate $I_{\mathrm{VBM}}$ compared to experiment-a well known artifact of the inability of GGA to exclude electron self-interaction with sufficient accuracy $[67,68]$.

The use of nonlocal XC functionals has been shown to be an effective method to counteract the inadequacies of GGA functionals in representing electronic structure of ionic materials [54]. Unsurprisingly, the PBE0 results are qualitatively better than calculated for PBE, with underestimates for $I_{\mathrm{VBM}}^{\mathrm{b}}$ compared to experiment of 0.63 and $0.75 \mathrm{eV}$ for $\mathrm{MgO}$ and $\mathrm{CaO}$, respectively, while the $I_{\mathrm{VBM}}^{\mathrm{b}}$ of $\mathrm{SrO}$ is close to $I_{\text {expt }}$. We also calculated the $I_{\mathrm{VBM}}^{\mathrm{b}}$ for $\mathrm{CdO}$ as $5.69 \mathrm{eV}$ in this case, which is lower than our experimental estimates of $6.04-6.78 \mathrm{eV}$. This experimental estimate for $\mathrm{CdO}$ is obtained by combining band gap measurements $(0.84 \mathrm{eV})$ with the electron affinity, which range over 5.2-5.94 eV [64-66]. Again, the results are 


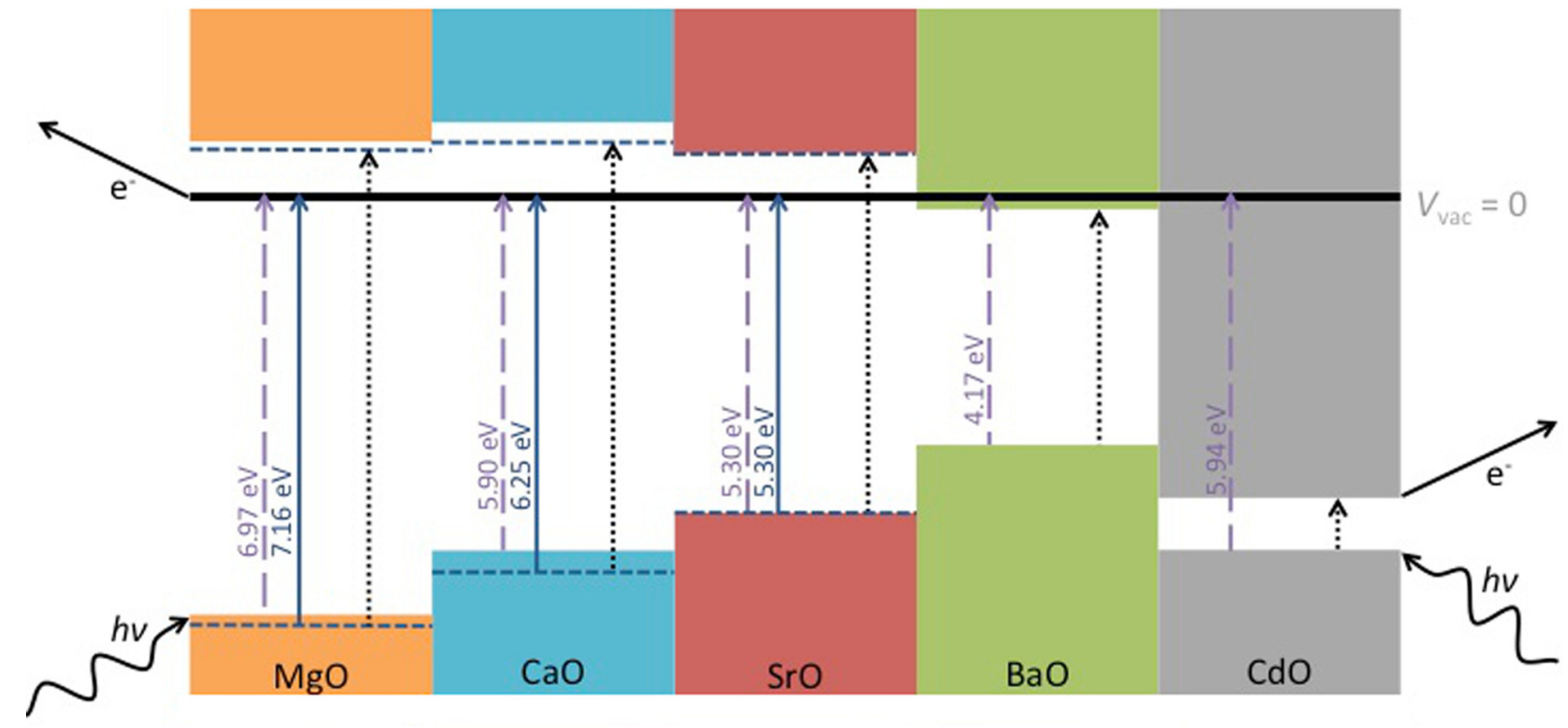

FIG. 3. (Color online) Schematic illustration of the ionization potential $I_{\mathrm{VBM}, \mathrm{D}}^{\mathrm{b}}$ for $\mathrm{MgO}$ (orange), $\mathrm{CaO}$ (blue), $\mathrm{SrO}$ (pink), $\mathrm{BaO}$ (green), and $\mathrm{CdO}$ (gray), as calculated using the PBESol0 XC functionals. Vacuum alignments were calculated using $I_{\mathrm{c}, \mathrm{D}}$ in Eq. (9) and illustrated with purple dashed lines. Experimental first ionization potentials $\left(I_{\text {expt }}\right)$ are also illustrated with blue arrows, and dashed blue horizontal markers. Black dotted lines mark out the experimental band gaps [64,69] and thus the position of the conduction band minimum relative to the vacuum is also illustrated. Experimental band gaps are chosen due to the uncertainty in the position of unoccupied orbitals at the DFT level, with calculated band gaps compared in the Appendices.

universally improved for $I_{\mathrm{VBM}, \mathrm{D}}^{\mathrm{b}}$ : the $I_{\mathrm{VBM}, \mathrm{D}}^{\mathrm{b}}$ of $\mathrm{MgO}$ and $\mathrm{CaO}$ are now only underestimated by 0.27 and $0.58 \mathrm{eV}$, respectively, and the $I_{\mathrm{VBM}, \mathrm{D}}^{\mathrm{b}}$ of $\mathrm{SrO}$ remains relatively constant with a difference of only $0.09 \mathrm{eV}$ compared to $I_{\text {expt }}$. Furthermore, the $I_{\mathrm{VBM}, \mathrm{D}}^{\mathrm{b}}$ of CdO is increased by $0.66 \mathrm{eV}$, to $6.35 \mathrm{eV}$, much closer to our experimental estimate.

Finally, we repeated all our calculations using the PBESol0 $\mathrm{XC}$ functional due to its success in reproducing bulk properties [70]. The resulting ionization potentials are all greater than those calculated using PBE0, in contrast to our observations for the GGA level of theory: the calculated $I_{\mathrm{VBM}, \mathrm{D}}^{\mathrm{b}}$ of $6.97,5.90$, and $5.30 \mathrm{eV}$ for $\mathrm{MgO}, \mathrm{CaO}$, and $\mathrm{SrO}$ deviate from experiment by only $0.19,0.35$, and $0.00 \mathrm{eV}$, respectively, as illustrated in Fig. 3. Special attention must be paid, however, to the position of the VBM of $\mathrm{CdO}$ in this case. When the vacuum level is aligned for PBESol0 using $I_{\mathrm{c}}$ [Eq. (3)], the VBM of $\mathrm{CdO}$ is positioned above both $\mathrm{CaO}$ and $\mathrm{SrO}$. In contrast, when using PBE0, this same method positions the VBM for CdO below the VBM of $\mathrm{CaO}$ and $\mathrm{SrO}$ (Fig. 3). Thus an obvious question exists: which alignment is correct? $I_{\mathrm{c}, \mathrm{D}}[\mathrm{Eq}$. (9)] gives a more consistent answer, as for both nonlocal $\mathrm{XC}$ functionals the VBM of $\mathrm{CdO}$ is positioned below that of $\mathrm{CaO}$ and $\mathrm{SrO}$.

Presented in Table II are $\Delta V_{\mathrm{bg}}^{\mathrm{s}}, \Delta V_{\mathrm{c}}^{\mathrm{s}}$, and $D_{\mathrm{s}}$, as calculated using the reparametrized shell model potentials, for local (PBESol) and nonlocal (PBESol0) XC functionals. The similarity of calculated values between both types of $\mathrm{XC}$ functional is evident. Furthermore, the trend in results can be convincingly linked to the increasing cation radius as one descends the group: $0.86 \AA$ (Mg), $1.14 \AA$ (Ca), $1.32 \AA$ ( $\mathrm{Sr})$, $1.49 \AA(\mathrm{Ba})$, and the polarizability of the ionic oxides (results included in the Appendices). We note that the overall sign of
$D_{\text {s }}$ correlates with the radius of the cation relative to the $\mathrm{O}$ anion, which has an ionic radius of $1.26 \AA$ [71].

For the smaller $\mathrm{Mg}$ and $\mathrm{Ca}$ cations, the total shift in electrostatic potential is large and negative, implying that electrons distribute inward from the slab surface. This results in an underestimate of the ionization potential. For the $\mathrm{Sr}$ and $\mathrm{Ba}$ cations, which are larger than $\mathrm{O}$ anions, the shift in $D_{\text {s }}$ is positive, highlighting electron accumulation towards the surface, though further verification is needed to study these effects for actual relaxed surfaces.

We note that the differences in the Madelung potential between equivalent sites in the bulk unit cell and the bulklike positions in the middle of the fixed slab supercell were used to give an accurate representation of the error bars in using the described "slab alignment" method. For the group 2 (12) metal oxides, the error bars are less than $\pm 0.02( \pm 0.14) \mathrm{V}$ before shell optimization.

TABLE II. Calculated shifts $\Delta V_{\mathrm{bg}}^{\mathrm{s}}$ and $\Delta V_{\mathrm{c}}^{\mathrm{s}}$, respectively, in $\mathrm{V}$, as a function of compound for the slab geometries as a result of shell relaxation. Results for the PBESol (PBESolO) XC functionals are presented. The combined polarization offset, $D_{\mathrm{s}}$, is given in $\mathrm{eV}$.

\begin{tabular}{lccccc}
\hline \hline & $\mathrm{MgO}$ & $\mathrm{CaO}$ & $\mathrm{SrO}$ & $\mathrm{BaO}$ & $\mathrm{CdO}$ \\
\hline$\Delta V_{\mathrm{bg}}^{\mathrm{s}}$ & 0.45 & 0.21 & 0.10 & -0.14 & \\
& $(0.40)$ & $(0.10)$ & $(-0.02)$ & $(-0.29)$ & $(0.51)$ \\
$\Delta V_{\mathrm{c}}^{\mathrm{s}}$ & -0.53 & -0.18 & -0.05 & 0.20 & \\
& $(-0.46)$ & $(-0.07)$ & $(0.06)$ & $(0.34)$ & $(-0.15)$ \\
$D_{\mathrm{s}}$ & 0.98 & 0.39 & 0.16 & -0.34 & \\
& $(0.86)$ & $(0.16)$ & $(-0.08)$ & $(-0.63)$ & $(0.66)$ \\
\hline \hline
\end{tabular}




\section{CONCLUSIONS}

In conclusion, we have investigated the slab model technique for vacuum alignment of energy levels in bulk materials at the plane-wave DFT level, and illustrated deficiencies in the application of this method. Explicit consideration of the electronic polarization, induced by the presence of a surface, is necessary for accurate results. We have proposed a method for quantifying the electronic polarization using simple polarizable shell-based interatomic potentials that have been tuned to reproduce accurately the properties predicted by DFT calculations. Our updated results for rocksalt ionic oxides using nonlocal $\mathrm{XC}$ functionals show excellent agreement with the available experimental literature when using our suggested bulk alignment protocol, illustrating the potential of this method. Our observations open a path to understanding the extent of fundamental band bending: knowledge of the bulk ionization potential would allow for the crystal engineering of band alignments for specific applications using band bending effects. Further work is ongoing to generalize our observations for more complex materials, as well as consideration of surfaces with intrinsic polar moments such as the more complex polymorphs of common semiconducting oxides.

\section{ACKNOWLEDGMENTS}

The authors acknowledge stimulating discussions with Aron Walsh and Keith Butler. A.J.L., C.R.A.C., and A.A.S. acknowledge funding from EPSRC Grants No. EP/IO30662/1 and No. EP/K038419/1. The authors acknowledge the use of the following high-performance computing facilities, and associated support services, in the completion of this work: HECToR, via our membership of the UK HPC Materials Chemistry Consortium (EP/L000202), IRIDIS, provided by the Centre for Innovation, and UCL Legion.

\section{APPENDIX A: DFT OBSERVABLES: LATTICE PARAMETER, BAND GAP, AND POLARIZABILITY}

The calculated DFT observables, as used to reparameterize the interatomic potentials and calculate the position of the valence band maximum, are presented. Namely, the data presented are the lattice parameter of the structure (Table III), the electronic band gap (Table IV), and the high-frequency dielectric constant (Table V).

TABLE III. DFT-calculated lattice parameter, $a_{0}(\AA)$, for the cubic unit cells of $\mathrm{MgO}, \mathrm{CaO}, \mathrm{SrO}, \mathrm{BaO}$, and $\mathrm{CdO}$, as optimized using the analytical stress tensor. Calculations were performed using the PBE, PBESol, PBE0, and PBESol0 exchange-correlation functionals, respectively. For comparison, experimental measurements from the literature at the lowest temperature available are also presented, with the temperature $(\mathrm{K})$ given in parentheses [72-75].

\begin{tabular}{lccccc}
\hline \hline & Expt. & PBE & PBESol & PBE0 & PBESol0 \\
\hline $\mathrm{MgO}$ & $4.21(19.8)$ & 4.26 & 4.22 & 4.21 & 4.18 \\
$\mathrm{CaO}$ & $4.80(17.9)$ & 4.84 & 4.78 & 4.80 & 4.77 \\
$\mathrm{SrO}$ & $5.16(293)$ & 5.19 & 5.14 & 5.17 & 5.12 \\
$\mathrm{BaO}$ & $5.54(298)$ & 5.61 & 5.53 & 5.56 & 5.52 \\
$\mathrm{CdO}$ & $4.70(294)$ & & & 4.72 & 4.67 \\
\hline \hline
\end{tabular}

TABLE IV. DFT-calculated band gap, $E_{\mathrm{g}}(\mathrm{eV})$, for the optimized cubic unit cells of $\mathrm{MgO}(\Gamma-\Gamma), \mathrm{CaO}(\Gamma-M), \mathrm{SrO}(\Gamma-M), \mathrm{BaO}$ $(M-M)$, and $\mathrm{CdO}(X-\Gamma)$. Calculations were performed using the PBE, PBESol, PBE0, and PBESol0 exchange-correlation functionals, respectively. For comparison, direct experimental measurements from the literature at the lowest temperature available are also presented (indirect for $\mathrm{CdO}$; direct for all other materials), with the temperature (K) given in parentheses $[64,69,76]$.

\begin{tabular}{lccccc}
\hline \hline & Expt. & PBE & PBESol & PBE0 & PBESol0 \\
\hline $\mathrm{MgO}$ & $7.83(85)$ & 4.45 & 4.61 & 7.24 & 7.38 \\
$\mathrm{CaO}$ & $7.09(85)$ & 3.63 & 3.52 & 6.04 & 5.94 \\
$\mathrm{SrO}$ & $5.90(55)$ & 3.27 & 3.75 & 5.47 & 5.37 \\
$\mathrm{BaO}$ & $3.89(55)$ & 3.27 & 3.29 & 4.01 & 3.84 \\
$\mathrm{CdO}$ & $0.85(100)$ & & & 1.56 & 1.55 \\
\hline \hline
\end{tabular}

TABLE V. DFT-calculated high-frequency dielectric constant, $\varepsilon(\infty)$, for the optimized cubic unit cells of $\mathrm{MgO}, \mathrm{CaO}, \mathrm{SrO}, \mathrm{BaO}$, and $\mathrm{CdO}$. Calculations were performed using the PBE, PBESol, PBE0, and PBESol0 exchange-correlation functionals, respectively. For comparison, experimental measurements from the literature are also presented [77-80].

\begin{tabular}{lccccc}
\hline \hline & Expt. & PBE & PBESol & PBE0 & PBESol0 \\
\hline $\mathrm{MgO}$ & 2.96 & 3.26 & 3.33 & 2.61 & 2.63 \\
$\mathrm{CaO}$ & 3.33 & 3.91 & 4.01 & 2.99 & 3.03 \\
$\mathrm{SrO}$ & 3.46 & 3.95 & 4.07 & 3.03 & 3.09 \\
$\mathrm{BaO}$ & 3.90 & 4.46 & 4.62 & 3.31 & 3.38 \\
$\mathrm{CdO}$ & 5.3 & & & 4.01 & 4.05 \\
\hline \hline
\end{tabular}

TABLE VI. Parameters for optimized interatomic potentials of $\mathrm{MgO}, \mathrm{CaO}, \mathrm{SrO}, \mathrm{BaO}$, and $\mathrm{CdO}$, respectively. The interaction parameter, $A(\mathrm{eV})$, between the cation and anion shells in each system, and the anion shell spring constant, $k\left(\mathrm{eV}^{-2}\right)$, have been tuned to reproduce $a_{0}$ and $\varepsilon(\infty)$ from DFT calculations, as performed using the PBE, PBESol, PBE0, and PBESol0 exchange-correlation functionals, respectively. All other components of the interatomic potentials were unaltered from the original work of Lewis and Catlow [57]. For the $\mathrm{CdO}$ system, the $\mathrm{Cd}^{2+}$ cation was modeled as an unpolarizable species, due to the absence in the literature of a completely polarizable potential for this system. In all cases the observables calculated using the tuned interatomic potential were within $0.0001 \%$ of the same DFT observables after fitting.

\begin{tabular}{lcrrrr}
\hline \hline & & PBE & PBESol & PBE0 & PBESol0 \\
\hline $\mathrm{MgO}$ & $A(\mathrm{Mg}-\mathrm{O})$ & 1517.21 & 1447.76 & 1431.20 & 1383.76 \\
& $k(\mathrm{O})$ & 47.59 & 48.08 & 66.39 & 66.99 \\
$\mathrm{CaO}$ & $A(\mathrm{Ca}-\mathrm{O})$ & 1124.32 & 1061.06 & 1085.33 & 1042.17 \\
& $k(\mathrm{O})$ & 37.08 & 37.97 & 58.98 & 60.30 \\
$\mathrm{SrO}$ & $A(\mathrm{Sr}-\mathrm{O})$ & 996.37 & 944.34 & 969.30 & 921.57 \\
& $k(\mathrm{O})$ & 34.68 & 35.33 & 58.97 & 60.37 \\
$\mathrm{BaO}$ & $A(\mathrm{Ba}-\mathrm{O})$ & 984.53 & 917.18 & 941.69 & 907.33 \\
& $k(\mathrm{O})$ & 30.76 & 32.06 & 55.06 & 55.83 \\
$\mathrm{CdO}$ & $A(\mathrm{Cd}-\mathrm{O})$ & & & 870.82 & 827.33 \\
& $k(\mathrm{O})$ & & & 29.9196 & 30.4693 \\
\hline \hline
\end{tabular}




\section{APPENDIX B: FORCEFIELD PARAMETERS: SHELL MODEL}

The optimized interatomic potentials, as used in the calculation of the surface polarization effects, are presented in Table VI for the rocksalt structured systems of interest: $\mathrm{MgO}, \mathrm{CaO}, \mathrm{SrO}, \mathrm{BaO}$ and $\mathrm{CdO}$.
[1] A. Franciosi and C. G. V. de Walle, Surf. Sci. Rep. 25, 1 (1996).

[2] S. F. J. Cox, J. L. Gavartin, J. S. Lord, S. P. Cottrell, J. M. Gil, H. V. Alberto, J. P. Duarte, R. C. Vilão, N. A. de Campos, D. J. Keeble, E. A. Davis, M. Charlton, and D. P. van der Werf, J. Phys.: Condens. Matter 18, 1079 (2006).

[3] W. R. Frensley and H. Kroemer, J. Vac. Sci. Technol. 13, 810 (1976).

[4] W. R. Frensley and H. Kroemer, Phys. Rev. B 16, 2642 (1977).

[5] W. A. Harrison, J. Vac. Sci. Technol. 14, 1016 (1977).

[6] W. A. Harrison, J. Vac. Sci. Technol. B 3, 1231 (1985).

[7] D. O. Scanlon, C. W. Dunnill, J. Buckeridge, S. A. Shevlin, A. J. Logsdail, S. M. Woodley, C. R. A. Catlow, M. J. Powell, R. G. Palgrave, I. P. Parkin, G. W. Watson, T. W. Keal, P. Sherwood, A. Walsh, and A. A. Sokol, Nat. Mater. 12, 798 (2013).

[8] C. G. Van de Walle and R. M. Martin, in Electronic Structure of Semiconductor Heterojunctions, edited by G. Margaritondo, Perspectives in Condensed Matter Physics Vol. 1 (Springer, Netherlands, 1988), pp. 268-279.

[9] C. G. Van de Walle and J. Neugebauer, Nature (London) 423, 626 (2003).

[10] Y.-H. Li, A. Walsh, S. Chen, W.-J. Yin, J.-H. Yang, J. Li, J. L. Da Silva, X. Gong, and S.-H. Wei, Appl. Phys. Lett. 94, 212109 (2009).

[11] S. B. Zhang, S.-H. Wei, and A. Zunger, Phys. Rev. Lett. 84, 1232 (2000).

[12] C. Kilic and A. Zunger, Appl. Phys. Lett. 81, 73 (2002).

[13] J. Tersoff, Phys. Rev. B 30, 4874 (1984).

[14] F. Flores and C. Tejedor, J. Phys. C: Solid State 12, 731 (1979).

[15] W. Mönch, J. Appl. Phys. 109, 113724 (2011).

[16] F. Jensen, Introduction to Computational Chemistry (Wiley, Chichester, 1999).

[17] P. Hohenberg and W. Kohn, Phys. Rev. 136, B864 (1964).

[18] W. Kohn and L. J. Sham, Phys. Rev. 140, A1133 (1965).

[19] P. P. Ewald, Ann. Phys. 369, 253 (1921).

[20] A. Baldereschi, S. Baroni, and R. Resta, Phys. Rev. Lett. 61, 734 (1988).

[21] Y. Z. Zhu, G. D. Chen, H. Ye, A. Walsh, C. Y. Moon, and S.-H. Wei, Phys. Rev. B 77, 245209 (2008).

[22] A. Janotti and C. G. Van de Walle, Phys. Rev. B 75, 121201 (2007).

[23] S.-H. Wei and A. Zunger, Appl. Phys. Lett. 72, 2011 (1998).

[24] R. Dovesi, R. Orlando, B. Civalleri, C. Roetti, V. R. Saunders, and C. M. Zicovich-Wilson, Z. Kristallogr. 220, 571 (2005).

[25] R. Car and M. Parrinello, Phys. Rev. Lett. 55, 2471 (1985).

[26] M. D. Segall, P. J. D. Lindan, M. J. Probert, C. J. Pickard, P. J. Hasnip, S. J. Clark, and M. C. Payne, J. Phys.: Condens. Matter 14, 2717 (2002)

[27] G. Kresse and J. Furthmüller, Comput. Mater. Sci. 6, 15 (1996).

[28] G. Kresse and D. Joubert, Phys. Rev. B 59, 1758 (1999).

[29] B. Höffling, A. Schleife, F. Fuchs, C. Rödl, and F. Bechstedt, Appl. Phys. Lett. 97, 032116 (2010).
[30] M. C. Toroker, D. K. Kanan, N. Alidoust, L. Y. Isseroff, P. Liao, and E. A. Carter, Phys. Chem. Chem. Phys. 13, 16644 (2011).

[31] D. West, Y. Y. Sun, and S. B. Zhang, Appl. Phys. Lett. 101, 082105 (2012).

[32] B. Höffling, A. Schleife, C. Rödl, and F. Bechstedt, Phys. Rev. B 85, 035305 (2012).

[33] L. A. Burton and A. Walsh, Appl. Phys. Lett. 102, 13211 (2013).

[34] A. Walsh and C. R. A. Catlow, J. Mater. Chem. 20, 10438 (2010).

[35] J. Carrasco, F. Illas, and N. Lopez, Phys. Rev. Lett. 100, 016101 (2008).

[36] L. B. Vilhelmsen and B. Hammer, Phys. Rev. Lett. 108, 126101 (2012).

[37] N. A. Richter, S. Sicolo, S. V. Levchenko, J. Sauer, and M. Scheffler, Phys. Rev. Lett. 111, 045502 (2013).

[38] P. Rinke, A. Schleife, E. Kioupakis, A. Janotti, C. Rödl, F. Bechstedt, M. Scheffler, and C. G. Van de Walle, Phys. Rev. Lett. 108, 126404 (2012).

[39] B. Ulrici, W. Ulrici, and N. N. Kovalev, Fiz. Tverd. Tela 17, 3533 (1975) [Sov. Phys. Solid State 17, 2305 (1975)].

[40] P. Broqvist, H. Grönbeck, and I. Panas, Surf. Sci. 554, 262 (2004).

[41] T. Koopmans, Physica 1, 104 (1934).

[42] E. J. Baerends, O. V. Gritsenko, and R. van Meer, Phys. Chem. Chem. Phys. 15, 16408 (2013).

[43] A. Walsh and K. T. Butler, Acc. Chem. Res. 47, 364 (2014).

[44] K. T. Butler, J. Buckeridge, C. R. A. Catlow, and A. Walsh, Phys. Rev. B 89, 115320 (2014).

[45] Z. Zhang and J. T. Yates, Chem. Rev. 112, 5520 (2012).

[46] K. T. Butler and A. Walsh, Thin Solid Films 559, 64 (2014).

[47] L. Guimarães, H. A. de Abreu, and H. A. Duarte, Chem. Phys. 333, 10 (2007).

[48] P. E. Blöchl, Phys. Rev. B 50, 17953 (1994).

[49] H. J. Monkhorst and J. D. Pack, Phys. Rev. B 13, 5188 (1976).

[50] G. P. Francis and M. C. Payne, J. Phys.: Condens. Matter 2, 4395 (1990).

[51] J. P. Perdew, K. Burke, and M. Ernzerhof, Phys. Rev. Lett. 77, 3865 (1996).

[52] J. P. Perdew, A. Ruzsinszky, G. I. Csonka, O. A. Vydrov, G. E. Scuseria, L. A. Constantin, X. Zhou, and K. Burke, Phys. Rev. Lett. 100, 136406 (2008).

[53] C. Adamo and V. Barone, J. Chem. Phys. 110, 6158 (1999).

[54] M. Marsman, J. Paier, A. Stroppa, and G. Kresse, J. Phys.: Condens. Matter 20, 064201 (2008).

[55] M. Gajdoš, K. Hummer, G. Kresse, J. Furthmüller, and F. Bechstedt, Phys. Rev. B 73, 045112 (2006).

[56] P. W. Tasker, J. Phys. C: Solid State 12, 4977 (1979).

[57] G. V. Lewis and C. R. A. Catlow, J. Phys. C: Solid State 18, 1149 (1985).

[58] J. D. Gale and A. L. Rohl, Mol. Simul. 29, 291 (2003). 
[59] L. Pauling, Proc. R. Soc. A 114, 181 (1927).

[60] E. D. Palik, Handbook of Optical Constants of Solids (Academic Press, New York, 1985).

[61] P. D. Johnson, Phys. Rev. 94, 845 (1954).

[62] V. I. Neeley and J. C. Kemp, J. Phys. Chem. Solids 24, 1301 (1963).

[63] T. Jaouen, G. Jézéquel, G. Delhaye, B. Lépine, P. Turban, and P. Schieffer, Appl. Phys. Lett. 97, 232104 (2010).

[64] F. P. Koffyberg, Phys. Rev. B 13, 4470 (1976).

[65] W.-M. Cho, G.-R. He, T.-H. Su, and Y.-J. Lin, Appl. Surf. Sci. 258, 4632 (2012).

[66] D. Speaks, M. Mayer, K. Yu, S. Mao, E. Haller, and W. Walukiewicz, J. Appl. Phys. 107, 113706 (2010).

[67] J. P. Perdew and M. Levy, Phys. Rev. Lett. 51, 1884 (1983).

[68] L. J. Sham and M. Schlüter, Phys. Rev. Lett. 51, 1888 (1983).

[69] A. S. Rao and R. J. Kearney, Physica Status Solidi (b) 95, 243 (1979).
[70] Chemical Modelling, edited by M. Springborg, SPR-Chemical Modelling Vol. 9 (The Royal Society of Chemistry, Cambridge, UK, 2012), pp. P001-217.

[71] R. D. Shannon, Acta Crystallogr. 32, 751 (1976).

[72] D. K. Smith and H. R. Leider, J. Appl. Crystallogr. 1, 246 (1968).

[73] J. Bashir, R. T. A. Khan, N. M. Butt, and G. Heger, Powder Diffr. 17, 222 (2002).

[74] R. J. Zollweg, Phys. Rev. 100, 671 (1955).

[75] A. Cimino and M. Marezio, J. Phys. Chem. Solids 17, 57 (1960).

[76] R. Whited, C. J. Flaten, and W. C. Walker, Solid State Commun. 13, 1903 (1973).

[77] R. E. Stephens and I. H. Malitson, J. Res. Natl. Bur. Stand. (US) 49, 249 (1952).

[78] J. Jacobson and E. Nixon, J. Phys. Chem. Solids 29, 967 (1968).

[79] C. J. Anderson and E. B. Hensley, J. Appl. Phys. 46, 443 (1975).

[80] H. Finkenrath, H. Köhler, and M. Lochmann, Z. Ang. Phys. 21, 512 (1966). 